\title{
Minimum Cost Homomorphisms to Semicomplete Bipartite Digraphs
}

\author{
Gregory Gutin* Arash Rafiey ${ }^{\dagger} \quad$ Anders $\mathrm{Yeo}^{\ddagger}$
}

\begin{abstract}
For digraphs $D$ and $H$, a mapping $f: V(D) \rightarrow V(H)$ is a homomorphism of $D$ to $H$ if $u v \in A(D)$ implies $f(u) f(v) \in A(H)$. If, moreover, each vertex $u \in V(D)$ is associated with costs $c_{i}(u), i \in V(H)$, then the cost of the homomorphism $f$ is $\sum_{u \in V(D)} c_{f(u)}(u)$. For each fixed digraph $H$, we have the minimum cost homomorphism problem for $H$. The problem is to decide, for an input graph $D$ with costs $c_{i}(u)$, $u \in V(D), i \in V(H)$, whether there exists a homomorphism of $D$ to $H$ and, if one exists, to find one of minimum cost. Minimum cost homomorphism problems encompass (or are related to) many well studied optimization problems. We describe a dichotomy of the minimum cost homomorphism problem for semicomplete multipartite digraphs $H$. This solves an open problem from an earlier paper. To obtain the dichotomy of this paper, we introduce and study a new notion, a $k$-Min-Max ordering of digraphs.
\end{abstract}

\section{Introduction}

Motivation. We consider only directed (undirected) graphs that have neither loops nor multiple arcs (edges). In this paper we solve a problem raised in [5] to find a dichotomy for the computational complexity of minimum cost homomorphism problem (MCH) for semicomplete bipartite digraphs (we define this problem below). In fact, our result leads to a complete dichotomy for the computational complexity of $\mathrm{MCH}$ for semicomplete $k$ partite digraphs $(k \geq 2)$ as a (much simpler) dichotomy for the case $k \geq 3$ was obtained in [5] (see also Section 5). Our result uses and significantly extends a dichotomy for the computational complexity of $\mathrm{MCH}$ for bipartite undirected graphs obtained in [3].

\footnotetext{
${ }^{*}$ Corresponding author. Department of Computer Science, Royal Holloway University of London, Egham, Surrey TW20 OEX, UK, gutin@cs.rhul.ac.uk

${ }^{\dagger}$ School of Computing Science, Simon Fraser University, Burnaby, B.C., Canada, V5A 1S6, arafieyh@cs.sfu.ca

${ }^{\ddagger}$ Department of Computer Science, Royal Holloway University of London, Egham, Surrey TW20 OEX, UK, anders@cs.rhul.ac.uk
} 
In our previous papers we used properties of an important notion of Min-Max ordering of digraphs. To obtain the dichotomy of this paper, we introduce and study a new notion, a $k$-Min-Max ordering of digraphs. We believe that properties of this notion and, in particular, Theorem 2.2 can be used to obtain further results on $\mathrm{MCH}$ and its special cases (see below).

The minimum cost homomorphism problem was introduced in [6], where it was motivated by a real-world problem in defence logistics. We believe it offers a practical and natural model for optimization of weighted homomorphisms. MCH's special cases include the well-known list homomorphism problem [8, 10, and the general optimum cost chromatic partition problem, which has been intensively studied [7, 11, 12, and has a number of applications, 14, 15.

Minimum cost homomorphisms. For directed or undirected graphs $G$ and $H$, a mapping $f: V(G) \rightarrow V(H)$ is a homomorphism of $G$ to $H$ if $u v \in E(G)$ implies $f(u) f(v) \in$ $E(H)$. Recent treatments of homomorphisms in directed and undirected graphs can be found in [8, 10]. Let $H$ be a fixed directed or undirected graph. The homomorphism problem for $H$ asks whether a directed or undirected input graph $G$ admits a homomorphism to $H$. The list homomorphism problem for $H$ asks whether a directed or undirected input graph $G$ with lists (sets) $L_{u} \subseteq V(H), u \in V(G)$ admits a homomorphism $f$ to $H$ in which $f(u) \in L_{u}$ for each $u \in V(G)$.

Suppose $G$ and $H$ are directed (or undirected) graphs, and $c_{i}(u), u \in V(G), i \in V(H)$ are nonnegative costs. The cost of a homomorphism $f$ of $G$ to $H$ is $\sum_{u \in V(G)} c_{f(u)}(u)$. If $H$ is fixed, the minimum cost homomorphism problem, $\operatorname{MinHOM}(H)$, for $H$ is the following optimization problem. Given an input graph $G$, together with costs $c_{i}(u), u \in V(G)$, $i \in V(H)$, we wish to find a minimum cost homomorphism of $G$ to $H$, or state that none exists.

A bipartite digraph is semicomplete if there is at least one arc between every two vertices belonging to different partite sets. In this paper, we study the minimum cost homomorphism problem for semicomplete bipartite digraphs, i.e., $\operatorname{MinHOM}(H)$ when $H$ is a semicomplete bipartite digraph. Observe that $\mathrm{MCH}$ for semicomplete bipartite digraphs extends $\mathrm{MCH}$ for bipartite undirected graphs. Indeed, let $B$ be a semicomplete bipartite digraph with partite sets $U, V$ and arc set $A(B)=A_{1} \cup A_{2}$, where $A_{1}=\{u v: u \in$ $U, v \in V\}$ and $A_{2} \subseteq\{v u: v \in V, u \in U\}$. Let $B^{\prime}$ be a bipartite graph with partite sets $U, V$ and edge set $E\left(B^{\prime}\right)=\left\{u v: v u \in A_{2}\right\}$. Notice that $\operatorname{MinHOMP}(B)$ is equivalent to $\operatorname{MinHOMP}\left(B^{\prime}\right)$.

Min-Max ordering. Let $H$ be a digraph. We say that an ordering $v_{1}, v_{2}, \ldots, v_{p}$ of $V(H)$ is a Min-Max ordering of $H$ if $v_{i} v_{r}, v_{j} v_{s} \in A(H)$ implies $v_{\min \{i, j\}} v_{\min \{s, r\}} \in A(H)$ and $v_{\max \{i, j\}} v_{\max \{s, r\}} \in A(H)$. One can easily see that $v_{1}, v_{2}, \ldots, v_{p}$ of $V(H)$ is a Min-Max ordering of $H$ if $i<j, s<r$ and $v_{i} v_{r}, v_{j} v_{s} \in A(H)$, then $v_{i} v_{s} \in A(H)$ and $v_{j} v_{r} \in A(H)$. We can define a Min-Max ordering for a bipartite undirected graph $G$ with partite sets 


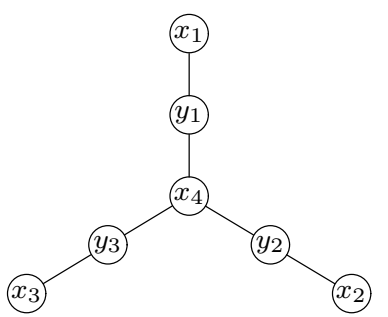

(a)

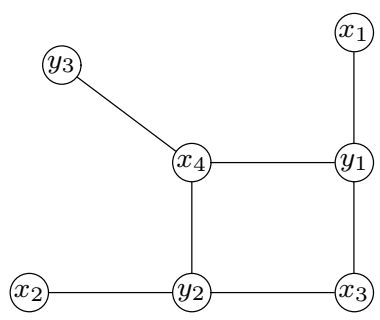

(b)

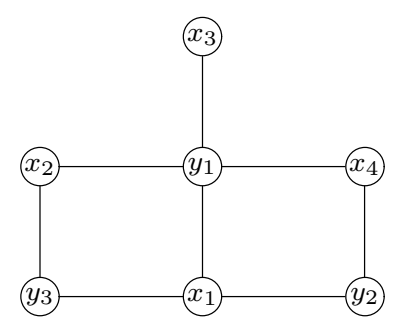

(c)

Figure 1: A bipartite claw (a), a bipartite net (b) and a bipartite tent (c).

$V$ and $U$ as follows: We orient all edges from $V$ to $U$ and apply the above definition for digraphs. Importance of Min-Max ordering for $\operatorname{MinHOM}(H)$ is indicated in the following two theorems.

Theorem 1.1 [4] Let a digraph $H$ have a Min-Max ordering. Then $\operatorname{MinHOM}(H)$ is polynomial-time solvable.

A bipartite graph $H$ with vertices $x_{1}, x_{2}, x_{3}, x_{4}, y_{1}, y_{2}, y_{3}$ is called

a bipartite claw if its edge set $E(H)=\left\{x_{4} y_{1}, y_{1} x_{1}, x_{4} y_{2}, y_{2} x_{2}, x_{4} y_{3}, y_{3} x_{3}\right\}$;

a bipartite net if its edge set $E(H)=\left\{x_{1} y_{1}, y_{1} x_{3}, y_{1} x_{4}, x_{3} y_{2}, x_{4} y_{2}, y_{2} x_{2}, y_{3} x_{4}\right\}$;

a bipartite tent if its edge set $E(H)=\left\{x_{1} y_{1}, y_{1} x_{3}, y_{1} x_{4}, x_{3} y_{2}, x_{4} y_{2}, y_{2} x_{2}, y_{3} x_{4}\right\}$.

See Figure 1.

Theorem 1.2 [3] Let $H$ be an undirected bipartite graph. If $H$ contains a cycle $C_{2 k}$, $k \geq 3$ or a bipartite claw or a bipartite net or a bipartite tent as an induced subgraph, then $\operatorname{MinHOM}(H)$ is NP-hard.

Assume that $P \neq N P$. Then the following three assertions are equivalent:

(i) H has a Min-Max ordering;

(ii) $\operatorname{MinHOM}(H)$ is polynomial time solvable;

(iii) $H$ does not contain a cycle $C_{2 k}, k \geq 3$, a bipartite claw, a bipartite net, or a bipartite tent as an induced subgraph.

Additional terminology and notation. For a graph $H, V(H)$ and $E(H)$ denote its vertex and edge sets, respectively. For a digraph $H, V(H)$ and $A(H)$ denote its vertex 
and arc sets, respectively. For a pair $X, Y$ of vertex sets of a digraph $H,(X, Y)_{H}$ denotes the set of all arcs of the form $x y$, where $x \in X, y \in Y$. We omit the subscript when it is clear from the context. Also, $X \times Y=\{x y: x \in X, y \in Y\}$. For a set $X \subseteq V(H)$, let $N^{+}(X)=\{y: \exists x \in X$ with $x y \in A(H)\}$ and $N^{-}(X)=\{y: \exists x \in X$ with $y x \in A(H)\}$.

If $x y$ is an arc of a digraph $H$, we will say that $x$ dominates $y, y$ is dominated by $x$, $y$ is an out-neighbor of $x$, and $x$ is an in-neighbor of $y$. We also denote it by $x \rightarrow y$. For disjoint sets $X, Y \subseteq V(H), X \rightarrow Y$ means that $x \rightarrow y$ for each $x \in X$ and $y \in Y$.

An extension of a digraph $G$ is a digraph $D$ obtained from $G$ by replacing each vertex $u$ of $G$ by a set of independent vertices $u_{1}, u_{2}, \ldots, u_{n(u)}$ such that for a pair $u, v$ of vertices in $G, u_{i} \rightarrow v_{j}$ in $D$ if and only if $u \rightarrow v$ in $G$.

For a bipartite digraph $H=(V, U ; A)$, where $V$ and $U$ are its partite sets, $H \rightarrow$ is the subdigraph induced by all arcs directed from $V$ to $U, H^{\leftarrow}$ is the subdigraph induced by all arcs directed from $U$ to $V$, and $H^{\leftrightarrow}$ is the subdigraph induced by all 2-cycles of $H$, i.e., by the set $\{x y: x y \in A, y x \in A\}$. The converse of $H$ is the digraph obtained from $H$ by replacing every arc $x y$ with the arc $y x$.

We denote a directed cycle with $p$ vertices by $\vec{C}_{p}$. For a set $X$ of vertices of a digraph $H, D[X]$ denotes the subdigraph of $H$ induced by $X$. For a digraph $H, U N(H)$ denotes the underlying graph of $H$, i.e., an undirected graph obtained from $H$ by disregarding all orientations and deleting multiple edges.

A digraph $D$ is strong (or, strongly connected) if there is a directed path from $x$ to $y$ and a directed path from $y$ to $x$ for every pair $x, y$ of vertices of $D$.

Forbidden family. Let us introduced five special digraphs for which, as we will see later, the minimum homomorphism problem is NP-hard. The digraph $C_{4}^{\prime}$ has vertex set $\left\{x_{1}, x_{2}, y_{1}, y_{2}\right\}$ and arc set $\left\{x_{1} y_{1}, y_{1} x_{2}, x_{2} y_{2}, y_{2} x_{1}, y_{1} x_{1}\right\}$. The digraph $C_{4}^{\prime \prime}$ has the same vertex set, but its arc set is $A\left(C_{4}^{\prime}\right) \cup\left\{x_{2} y_{1}\right\}$. The digraph $H^{*}$ has vertex set $\left\{x_{1}, x_{2}, y_{1}, y_{2}, y_{3}\right\}$ and arc set

$$
\left\{x_{1} y_{1}, y_{1} x_{2}, x_{2} y_{2}, y_{2} x_{1}, x_{1} y_{3}, x_{2} y_{3}\right\} \text {. }
$$

Let $N_{1}$ be a digraph with $V\left(N_{1}\right)=\left\{x_{1}, x_{2}, x_{3}, y_{1}, y_{2}, y_{3}\right\}$ and

$$
A\left(N_{1}\right)=\left\{x_{1} y_{1}, y_{1} x_{1}, x_{2} y_{2}, y_{2} x_{2}, x_{3} y_{3}, y_{3} x_{3}, y_{1} x_{2}, y_{1} x_{3}, x_{1} y_{2}, x_{1} y_{3}, x_{3} y_{2}, x_{2} y_{3}\right\} .
$$

Let $N_{2}$ be a digraph with $V\left(N_{2}\right)=\left\{x_{1}, x_{2}, x_{3}, y_{1}, y_{2}, y_{3}\right\}$ and

$$
A\left(N_{2}\right)=\left\{x_{1} y_{1}, x_{2} y_{2}, y_{2} x_{2}, x_{3} y_{3}, y_{3} x_{3}, y_{1} x_{2}, y_{1} x_{3}, x_{1} y_{2}, x_{1} y_{3}, x_{3} y_{2}, x_{2} y_{3}\right\} .
$$

A digraph $H$ belongs to the family $\mathcal{H} \mathcal{F O} \mathcal{R B}$ if $H$ or its converse is isomorphic to one of the five digraphs above or $U N\left(H^{s}\right)$ is isomorphic to bipartite claw, bipartite net, bipartite tent or even cycle with at least 6 vertices, where $s \in\{\rightarrow, \leftarrow, \leftrightarrow\}$.

$k$-Min-Max ordering. A collection $V_{1}, V_{2}, \ldots V_{k}$ of subsets of a set $V$ is called a $k$ partition of $V$ if $V=V_{1} \cup V_{2} \cup \cdots \cup V_{k}, V_{i} \cap V_{j}=\emptyset$ provided $i \neq j$. 
Definition 1.3 Let $H=(V, A)$ be a digraph and let $k \geq 2$ be an integer. We say that $H$ has a $k$-Min-Max ordering if there is a k-partition of $V$ into subsets $V_{1}, V_{2}, \ldots V_{k}$ and there is an ordering $v_{1}^{i}, v_{2}^{i}, \ldots, v_{\ell(i)}^{i}$ of $V_{i}$ for each $i$ such that

(i) Every arc of $H$ is an $\left(V_{i}, V_{i+1}\right)$-arc for some $i \in\{1,2, \ldots, k\}$,

(ii) $v_{1}^{i}, v_{2}^{i}, \ldots, v_{\ell(i)}^{i} v_{1}^{i+1} v_{2}^{i+1}, \ldots, v_{\ell(i+1)}^{i+1}$ is a Min-Max ordering of the subdigraph $H\left[V_{i} \cup\right.$ $\left.V_{i+1}\right]$ for all $i \in\{1,2, \ldots, k\}$,

where all indices $i+1$ are taken modulo $k$.

Note that if $H$ is a strong digraph in which the greatest common divisor of all cycle lengths is $k$, then $V(H)$ has a $k$-partition, $k \geq 2$, satisfying (i) (see Theorem 10.5.1 in [1]). A simple example of a digraph having a $k$-Min-Max ordering is an extension of $\vec{C}_{k}$.

Dichotomy and paper organization. The main result of this paper is the following:

Theorem 1.4 Let $H$ be an semicomplete bipartite digraph. If $H$ contains a digraph from $\mathcal{H} \mathcal{F O R B}$ as an induced subdigraph, then $\operatorname{MinHOM}(H)$ is NP-hard.

Assume that $P \neq N P$. Then the following three assertions are equivalent:

(i) $\operatorname{MinHOM}(H)$ is polynomial time solvable;

(ii) $H$ does not contain a digraph from $\mathcal{H} \mathcal{F O R B}$ as an induced subdigraph;

(iii) Each component of $H$ has a $k$-Min-Max ordering for $k=2$ or 4 .

Theorem 1.4 follows from Corollaries 3.5 and 4.6

The rest of the paper is organized as follows. In Section 2 we study properties of $k$ Min-Max orderings. In Section 3, we prove polynomial cases of $\operatorname{MinHOM}(H)$ when $H$ is a semicomplete bipartite digraph. In Section 4 we establish NP-hard cases of the problem. In Section 5 we formulate a dichotomy for the computational complexity of $\operatorname{MinHOM}(H)$ when $H$ is a semicomplete multipartite digraph. Section 6 provides a short discussion of further research.

\section{Properties of $k$-Min-Max Orderings}

Digraphs having $k$-Min-Max ordering have a very special structure as described in the following lemma.

Lemma 2.1 If a strong digraph $H=(V, A)$ has a $k$-Min-Max ordering as described in Definition 1.3, then we can define $0 \leq L(i, j)<R(i, j) \leq \ell(j)+1$ for all $i$ and $j$ such that 
(a): $N^{+}\left(v_{i}^{j}\right)=\left\{v_{L(i, j)+1}^{j+1}, v_{L(i, j)+2}^{j+1}, \ldots, v_{R(i, j)-1}^{j+1}\right\}$, where all superscripts are taken modulo $k$;

(b): For all $j$ and $i<i^{\prime}$ we have $R(i, j) \leq R\left(i^{\prime}, j\right)$ and $L(i, j) \leq L\left(i^{\prime}, j\right)$.

Proof: Suppose that $v_{i}^{j} v_{m-1}^{j+1}, v_{i}^{j} v_{m+1}^{j+1} \in A$, but $v_{i}^{j} v_{m}^{j+1} \notin A$. Since $H$ is strong, there is an arc $v_{t}^{j} v_{m}^{j+1}$ in $H$. By the definition of a $k$-Min-Max ordering, $v_{i}^{j} v_{m}^{j+1} \in A$, a contradiction that proves (a). Similarly, one can show (b).

The construction used in the following theorem was inspired by somewhat similar constructions in [13] and [2].

Theorem 2.2 If a digraph $H$ has a k-Min-Max ordering, then $\operatorname{MinHOM}(H)$ is polynomialtime solvable.

Proof: Let $V_{1}, V_{2}, \ldots, V_{k}$ be defined as in Definition 1.3 Let $D$ be an input digraph. Assume that there is homomorphism $f$ of $D$ to $H$. Let $G_{1}, G_{2}, \ldots, G_{k}$ be a $k$-partition of $V(D)$ such that $f\left(G_{j}\right) \subseteq V_{j}$ for each $j \in\{1,2, \ldots, k\}$. Observe that all arcs in $D$ are $\left(G_{j}, G_{j+1}\right)$-arcs, where all indices are taken modulo $k$ and $j \in\{1,2,3, \ldots, k\}$.

We will now show how to find a minimum cost homomorphism of $D$ to $H$, where the vertices of $G_{j}$ are mapped to $V_{j}$ for all $j=1,2, \ldots, k$. We will build a directed graph $\mathcal{L}$ with vertex set $\cup_{j=1}^{k}\left(G_{j} \times V_{j}\right)$ together with two other vertices, denoted by $s$ and $t$. We will also denote $t$ by $\left(x, v_{\ell(j)+1}^{j}\right)$ for every $j \in\{1,2, \ldots, k\}$. The weighted arcs of $\mathcal{L}$ are as follows, where $M$ is any constant greater than the cost of a minimum cost homomorphism of $D$ to $H$.

- An arc from $s$ to $\left(x, v_{1}^{j}\right)$, of weight $\infty$, for each $x \in G_{j}$.

- An arc from $\left(x, v_{i}^{j}\right)$ to $\left(x, v_{i+1}^{j}\right)$, of weight $c_{i}(x)+M$, for each $x \in G_{j}$ and $i \in$ $\{1,2, \ldots, \ell(j)\}$. Recall that when $i=\ell(j)$ the arc enters $t$.

- an arc from $\left(x, v_{i}^{j}\right)$ to $\left(y, v_{L(i, j)+1}^{j+1}\right)$ and an arc from $\left(y, v_{R(i, j)}^{j+1}\right)$ to $\left(x, v_{i+1}^{j}\right)$ for every $x y \in A(D)$ with $x \in G_{j}$ and every $i=1,2, \ldots, \ell(j)$. Furthermore the weight of these arcs are $\infty$.

A cut in $\mathcal{L}$ is a partition of the vertices into two sets $S$ and $T$ such that $s \in S$ and $t \in T$ and the weight of a cut is the sum of weights of all arcs going from a vertex of $S$ to a vertex of $T$. We will show that the minimum weight cut in $\mathcal{L}$ has weight equal to the minimum cost homomorphism of $D$ to $H$ plus $|V(D)| M$.

Let $f$ be a minimum cost homomorphism of $D$ to $H$, and assume that $f(x)=v_{a(x)}^{j}$ for each $x \in G_{j}$ and for all $j=1,2, \ldots, k$. Define a cut in $D$ as follows: $S=\left\{\left(x, v_{i}^{j}\right)\right.$ : 
$i \leq a(x), j=1,2, \ldots, k\} \cup\{s\}$ and $T=V(L)-S$. Note that the $\operatorname{arcs}$ from $\left(x, v_{a(x)}^{j}\right)$ to $\left(x, v_{a(x)+1}^{j}\right)$ belong to the cut and contribute $c_{f(x)}(x)+M$ to the weight of the cut. We will now show that there are no arcs of infinite weight in the cut, which would imply that the weight of $S$ is exactly the cost of a minimum cost homomorphism from $D$ to $H$ plus $|V(D)| M$

Clearly no arc out of $s$ belongs to the cut $S$. Assume for the sake of contradiction that the arc $\left(x, v_{i}^{j}\right)$ to $\left(y, v_{L(i, j)+1}^{j+1}\right)$ belongs to the cut $S$ for some $x y \in A(D)$ with $x \in G_{j}$. This implies that $a(x) \geq i\left(\operatorname{as}\left(x, v_{i}^{j}\right) \in S\right)$ and $a(y)<L(i, j)+1\left(\operatorname{as}\left(y, v_{L(i, j)+1}^{j+1}\right) \notin S\right)$. By Lemma 2.1 (b), this implies that $a(y) \leq L(i, j) \leq L(a(x), j)$. Thus, there is no arc from $v_{a(x)}^{j}$ to $v_{a(y)}^{j+1}$ in $H$, by the definition of $L(i, j)$. This is a contradiction to $f$ being a homomorphism.

Now assume for the sake of contradiction that the arc $\left(y, v_{R(i, j)}^{j+1}\right)$ to $\left(x, v_{i+1}^{j}\right)$ belongs to the cut $S$ for some $x y \in A(D)$ with $x \in G_{j}$. This implies that $a(x)<i+1$ (as $\left.\left(x, v_{i+1}^{j}\right) \notin S\right)$ and $a(y) \geq R(i, j)\left(\right.$ as $\left.\left(y, v_{R(i, j)}^{j+1}\right) \in S\right)$. By Lemma 2.1 (b), this implies that $a(y) \geq R(i, j) \geq R(a(x), j)$. Thus, there is no arc from $v_{a(x)}^{j}$ to $v_{a(y)}^{j+1}$ in $H$, by the definition of $R(i, j)$. This is a contradiction to $f$ being a homomorphism. We have now proved that the cut $S$ has the stated weight.

For the sake of contradiction assume that there exists a cut, $S^{\prime}$, in $\mathcal{L}$ of smaller weight than $S$. As the weight of $S$ is less than $M+|V(D)| M$ we note that the cut $S^{\prime}$ contains exactly one arc of the form $\left(x, v_{i}^{j}\right)\left(x, v_{i+1}^{j}\right)$ for each $x \in V(D)$. Therefore we may define a mapping, $f^{\prime}$, from $V(D)$ to $V(H)$ by letting $f^{\prime}(x)=v_{i}^{j}$ if and only if $\left(x, v_{i}^{j}\right) \in S^{\prime}$ and $\left(x, v_{i+1}^{j}\right) \notin S^{\prime}$. We will now show that $f^{\prime}$ is a homomorphism of $D$ to $H$ of smaller cost than $f$, a contradiction. This would imply that $S$ is a minimum weight cut, and we would be done.

Note that if $f^{\prime}$ is a homomorphism of $D$ to $H$, then it has smaller cost than $f$, as $S^{\prime}$ is a cut of smaller weight than $S$. Let $x y$ be any arc in $D$, and assume without loss of generality that $x \in G_{j}$. Let $i_{x}$ and $i_{y}$ be defined such that $\left(x, v_{i_{x}}^{j}\right) \in S^{\prime}$ and $\left(x, v_{i_{x}+1}^{j}\right) \notin S^{\prime}$ (i.e., $\left.f^{\prime}(x)=v_{i_{x}}^{j}\right)$ and $\left(y, v_{i_{y}}^{j+1}\right) \in S^{\prime}$ and $\left(y, v_{i_{y}+1}^{j+1}\right) \notin S^{\prime}$ (i.e., $\left.f^{\prime}(y)=v_{i_{y}}^{j+1}\right)$. As the arc $\left(x, v_{i_{x}}^{j}\right)\left(y, v_{L\left(i_{x}, j\right)+1}^{j+1}\right)$ is not in the cut, we must have $\left(y, v_{L\left(i_{x}, j\right)+1}^{j+1}\right) \in S$, which implies that $i_{y} \geq L\left(i_{x}, j\right)+1$. Furthermore as the arc $\left(y, v_{R\left(i_{x}, j\right)}^{j+1}\right)\left(x, v_{i_{x}+1}^{j}\right)$ is not in the cut, we must have $\left(y, v_{R\left(i_{x}, j\right)}^{j+1}\right) \notin S$, which implies that $i_{y}<R\left(i_{x}, j\right)$. We have now shown that $L\left(i_{x}, j\right)+1 \leq i_{y}<R\left(i_{x}, j\right)$, which by the definition of the functions $L$ and $R$ implies that $v_{i_{x}}^{j} v_{i_{y}}^{j+1}$ is an arc in $H$. Therefore $f^{\prime}$ is a homomorphism. 


\section{Polynomial Cases}

We start from a special case which is of importance when $H$ contains no induced $\vec{C}_{4}$.

Lemma 3.1 Let $H=(V, U ; A)$ be a semicomplete bipartite digraph, which does not contain an induced subdigraph belonging to $\mathcal{H} \mathcal{F O R B}$ or an induced directed 4-cycle. Suppose for every $v, v^{\prime} \in V$ we have $N^{+}(v) \subseteq N^{+}\left(v^{\prime}\right)$ or $N^{+}\left(v^{\prime}\right) \subseteq N^{+}(v)$. Then $H$ has a 2-MinMax ordering.

Proof: We say that vertices $v_{i}, v_{j} \in V$ are similar if $N^{+}\left(v_{i}\right)=N^{+}\left(v_{j}\right)$. Consider similarity classes $V_{1}, V_{2}, \ldots, V_{s}$ of $V$. Moreover assume that $N^{+}\left(V_{i}\right) \subset N^{+}\left(V_{j}\right)$ for $i<j$. Set $U_{1}=N^{+}\left(V_{1}\right)$ and $U_{i}=N^{+}\left(V_{i}\right)-\cup_{j=1}^{i-1} U_{j}$ for each $i>1$. If $s=1$ then $U N(H \rightarrow)$ is a complete bipartite graph and a Min-Max ordering of $H^{\leftarrow}$ (which exists by Theorem 1.2) is a 2-Min-Max ordering of $H$. Assume $s>1$. We prove the following two claims:

(1) Let $u_{i} \in U_{i}, v_{j} \in V_{j}, j>i$, and $u_{i} v_{j} \in A$. Then $U_{r} \rightarrow v_{j}$ for each $r>i$ and $u_{i} \rightarrow V_{t}$ for each $t<j$.

Proof of (1): By the definition of $U_{i}$ and $V_{i}$, if $r>j$ then $U_{r} \rightarrow v_{j}$. Now suppose that $i<r \leq j$ and $u_{r} v_{j} \notin A$ for some $u_{r} \in U_{r}$. Let $v_{i} \in V_{i}$ be arbitrary, and note that $H\left[\left\{u_{i}, v_{i}, u_{r}, v_{j}\right\}\right]$ is isomorphic to $C_{4}^{\prime}$ or $C_{4}^{\prime \prime}$, a contradiction. So $u_{r} v_{j} \in A$.

By the definition of $U_{i}$ and $V_{i}$, if $t<i$ then $u_{i} \rightarrow V_{t}$. Now suppose that $i \leq t<j$ and that $u_{i} v_{t} \notin A$, for some $v_{t} \in V_{t}$. Let $u_{j} \in U_{j}$ be arbitrary, and note that $H\left[\left\{u_{i}, v_{t}, u_{j}, v_{j}\right\}\right]$ is isomorphic to $C_{4}^{\prime}$ or $C_{4}^{\prime \prime}$, a contradiction.

(2) If $u, u^{\prime} \in U$ then $N^{+}(u) \subseteq N^{+}\left(u^{\prime}\right)$ or $N^{+}\left(u^{\prime}\right) \subseteq N^{+}(u)$.

Proof of (2): Assume that this is not the case, and there exist $v \in N^{+}(u)-N^{+}\left(u^{\prime}\right)$ and $v^{\prime} \in N^{+}\left(u^{\prime}\right)-N^{+}(u)$. This implies that $u \neq u^{\prime}, v \neq v^{\prime}, u v, u^{\prime} v^{\prime} \in A$ and $u v^{\prime}, u^{\prime} v \notin A$. If $u \in U_{i}$ and $u^{\prime} \in U_{j}$ and $i<j$, then by (1) we note that $u^{\prime} v \in A$ a contradiction. So for some $i$ we must have $\left\{u, u^{\prime}\right\} \subseteq U_{i}$. Analogously, if $v \in V_{a}$ and $v^{\prime} \in V_{b}$ and $a<b$, then by (1) we note that $u^{\prime} v \in A$, a contradiction. So for some $j$ we must have $\left\{v, v^{\prime}\right\} \subseteq V_{j}$. If $i>j$ then $U_{i} \rightarrow V_{j}$, which is a contradiction, so we must have $i \leq j$.

If $i<j$ then let $u^{\prime \prime} \in U_{j}$ and $v^{\prime \prime} \in V_{i}$ be arbitrary. Note that by (1) we must have the $\operatorname{arcs} u^{\prime \prime} v, u^{\prime \prime} v^{\prime}, u v^{\prime \prime}, u^{\prime} v^{\prime \prime}$ in $H$. By the construction of the sets $U_{i}, U_{j}, V_{i}, V_{j}$ we now note that the underlying graph of $H\left[\left\{u, u^{\prime}, u^{\prime \prime}, v, v^{\prime}, v^{\prime \prime}\right\}\right] \leftrightarrow$ is the 6 -cycle $v^{\prime \prime} u v u^{\prime \prime} v^{\prime} u^{\prime} v^{\prime \prime}$, a contradiction.

Therefore we must have $i=j$. First assume that $i<s$. Now consider $v_{s} \in V_{s}$ and $u_{s} \in U_{s}$. By (1) there is no arc from $\left\{u, u^{\prime}\right\}$ to $v_{s}$. However $H\left[\left\{u, u^{\prime}, v, v^{\prime}, v_{s}, u_{s}\right\}\right]$ is either $N_{1}$ or $N_{2}$, a contradiction. 
Similarly for the case $i=s$ we derive a contradiction.

Now consider an ordering $\left(u_{1}, u_{2}, \ldots, u_{a}\right)$ of the vertices in $U$ and an ordering $\left(v_{1}, v_{2}, \ldots, v_{b}\right)$ of the vertices in $V$, defined as follows. If $i<j$ then $d^{+}\left(v_{i}\right) \leq d^{+}\left(v_{j}\right)$ and if $d^{+}\left(v_{i}\right)=d^{+}\left(v_{j}\right)$ then $d^{-}\left(v_{i}\right) \geq d^{-}\left(v_{j}\right)$. Furthermore when $i<j$ then $d^{+}\left(u_{i}\right) \leq d^{+}\left(u_{j}\right)$ and if $d^{+}\left(u_{i}\right)=$ $d^{+}\left(u_{j}\right)$ then $d^{-}\left(u_{i}\right) \geq d^{-}\left(u_{j}\right)$. Note that the ordering $\left(v_{1}, v_{2}, \ldots, v_{b}\right)$ first contains vertices from $V_{1}$ then from $V_{2}$, etc.

We will now show that for every $i \in\{1,2, \ldots, b\}$ there exists an integer $\alpha_{i}$ such that $N^{+}\left(u_{i}\right)=\left\{v_{1}, v_{2}, \ldots, v_{\alpha_{i}}\right\}$. Suppose this is not the case. Then there exists an arc $u_{i} v_{j}$ in $H$, such that $u_{i} v_{j-1}$ is not an arc in $H$. Thus, both $v_{j}$ and $v_{j-1}$ belong to some $V_{k}$, as otherwise we have a contradiction to (1). This implies that $d^{+}\left(v_{j}\right)=d^{+}\left(v_{j-1}\right)$ and $d^{-}\left(v_{j-1}\right) \geq d^{-}\left(v_{j}\right)$. Note that every vertex $u \in N^{-}\left(v_{j-1}\right)$ has $N^{+}\left(u_{i}\right) \subseteq N^{+}(u)$, by (2). Therefore $u \rightarrow v_{j}$. However this implies that $d^{-}\left(v_{j-1}\right)<d^{-}\left(v_{j}\right)$ (as $u_{i} \rightarrow v_{j}$ but $u_{i}$ does not dominate $v_{j-1}$ ), a contradiction.

Using the fact that $N^{+}\left(u_{i}\right)=\left\{v_{1}, v_{2}, \ldots, v_{\alpha_{i}}\right\}$ for each $i \in\{1,2, \ldots, b\}$ and that $\alpha_{i} \geq \alpha_{i-1}$ for each $i \in\{2,3, \ldots, b\}$ (as $\left.d^{+}\left(u_{i}\right) \geq d^{+}\left(u_{i-1}\right)\right)$ and the similar relations for the vertices of $V$, we can readily conclude that $H$ has a 2-Min-Max ordering.

The distance $\operatorname{dist}(x, y)$ between a pair $x, y$ of vertices in an undirected graph $G$ is the length of the shortest path between $x$ and $y$. The diameter of $G$ is the maximal distance between a pair of vertices in $G$.

The following theorem shows when $\operatorname{MinHOM}(H)$ is polynomial time solvable if $H$ is strong and does not contain $\vec{C}_{4}$ as an induced subdigraph.

Theorem 3.2 Let $H$ be a strongly connected semicomplete bipartite digraph. Assume that $H$ does not contain a digraph from $\mathcal{H} \mathcal{F O} \mathcal{O R}$ or $\vec{C}_{4}$ as an induced subdigraph. Then $H$ has a 2-Min-Max ordering and $\operatorname{MinHOM}(H)$ is polynomial time solvable.

Furthermore, either $U N\left(H^{\rightarrow}\right)$ or $U N\left(H^{\leftarrow}\right)$ are complete bipartite graphs, or the following holds. For every pair $u, u^{\prime}$ of distinct vertices of $U$ we have $N^{+}(u) \subseteq N^{+}\left(u^{\prime}\right)$ or $N^{+}\left(u^{\prime}\right) \subseteq N^{+}(u)$ and for every pair $v, v^{\prime}$ of distinct vertices of $V$ we have $N^{+}(v) \subseteq N^{+}\left(v^{\prime}\right)$ or $N^{+}\left(v^{\prime}\right) \subseteq N^{+}(v)$.

Proof: By Theorem 2.2 to prove the first part part of this theorem (before 'Furthermore'), it suffices to show that $H$ has a 2-Min-Max ordering. Let $V$ and $U$ be partite sets of $H$. Denote $H_{1}=H^{\rightarrow}$ and $H_{2}=H^{\leftarrow}$. It follows from Theorem 1.2 that $U N\left(H_{1}\right)$ and $U N\left(H_{2}\right)$ have Min-Max orderings and so do $H_{1}$ and $H_{2}$.

Let $d_{i}$ be the diameter of $U N\left(H_{i}\right), i=1,2$. Observe that if $U N\left(H_{1}\right)\left(U N\left(H_{2}\right)\right)$ is a complete bipartite graph, then a Min-Max ordering of $H_{2}\left(H_{1}\right)$ is a 2-Min-Max ordering of $H$. Therefore, $\operatorname{MinHOM}(H)$ is polynomial time solvable by Theorem 2.2. Notice that 
$U N\left(H_{i}\right)$ is complete bipartite if and only if $d_{i}=2$. Thus, we may assume that both $d_{1} \geq 3$ and $d_{2} \geq 3$.

We consider the following cases for the value of $d_{1} \geq 3$.

Case 1: $d_{1}>4$.

We will show that $U N\left(H_{2}\right)$ is a complete bipartite graph or, equivalently, $d_{2}=2$. Assume that $d_{1}$ is odd, as the case of $d_{1}$ even can be considered similarly. Let $P=$ $v_{1} u_{1} v_{2} u_{2} \ldots v_{k-1} u_{k-1} v_{k} u_{k}$ be a shortest path of length $d_{1}$ between $v_{1}$ and $u_{k}$ in $U N\left(H_{1}\right)$. Let $\hat{U}=\left\{u_{1}, u_{2}, \ldots, u_{k}\right\}$ and $\hat{V}=\left\{v_{1}, v_{2}, \ldots, v_{k}\right\}$. We will first prove that $\hat{U} \rightarrow \hat{V}$. Since $P$ is a shortest path, we have $u_{i} \rightarrow v_{j}$ for each $u_{i} \in \hat{U}$ and $v_{j} \in \hat{V}$ provided $j \notin\{i, i+1\}$. Thus, it sufficient to prove

$$
u_{i} \rightarrow v_{i}(i=1,2, \ldots, k) \text { and } u_{i} \rightarrow v_{i+1}(i=1,2, \ldots, k-1)
$$

Consider the subdigraph $H^{\prime}$ of $H$ induced by four vertices $v_{i}, u_{i}, v_{i+2}, u_{i+1}$, where $i \in\{1,2, \ldots, k-2\}$. By the definition of $P$ (including the fact that $P$ is a shortest path), we have $v_{i} u_{i}, v_{i+2} u_{i+1}, u_{i+1} v_{i}, u_{i} v_{i+2} \in A(H)$, but $v_{i} u_{i+1}, v_{i+2} u_{i} \notin A(H)$. Since $H^{\prime}$ is not isomorphic to either $\vec{C}_{4}$ or $C_{4}^{\prime}$, we have $u_{i} v_{i}, u_{i+1} v_{i+2} \in A(H)$. This proves that $u_{i} \rightarrow v_{i}$ provided $i=1,2, \ldots, k-2$ and $u_{i} \rightarrow v_{i+1}$ provided $i=2,3, \ldots, k-1$. Thus, to prove (11) it remains to show that

$$
u_{k-1} \rightarrow v_{k-1}, u_{k} \rightarrow v_{k}, u_{1} \rightarrow v_{2}
$$

Consider the subdigraph $H^{\prime \prime}$ of $H$ induced by four vertices $v_{k-1}, u_{k-1}, v_{k}, u_{k}$. By the definition of $P$, we have $v_{k-1} u_{k-1}, v_{k} u_{k}, v_{k} u_{k-1}, u_{k} v_{k-1} \in A(H)$, but $v_{k-1} u_{k} \notin A(H)$. We have proved that $u_{k-1} \rightarrow v_{k}$. Since $H^{\prime \prime}$ is not isomorphic to $C_{4}^{\prime}$ or $C_{4}^{\prime \prime}$, we have $u_{k-1} v_{k-1}, u_{k} v_{k} \in A(H)$.

Consider $H\left[\left\{v_{2}, v_{3}, u_{1}, u_{2}\right\}\right]$. By the definition of $P$, we have $v_{2} u_{1}, v_{2} u_{2}, v_{3} u_{2}, u_{1} v_{3} \in$ $A(H)$, but $v_{3} u_{1} \notin A(H)$. We have proved that $u_{2} \rightarrow v_{2}$. Since $H\left[\left\{v_{2}, v_{3}, u_{1}, u_{2}\right\}\right]$ is not isomorphic to $C_{4}^{\prime}$ or $C_{4}^{\prime \prime}$, we have $u_{1} v_{2}, u_{2} v_{3} \in A(H)$. This implies that (2) and, thus, (11) has been proved.

Consider vertex $u \in U-\hat{U}$; we will show that $u \rightarrow\left\{v_{1}, v_{2}, \ldots, v_{k}\right\}$. Suppose this is not true. Let $j$ be the smallest index such that $u v_{j} \notin A(H)$. We have $v_{j} u \in A(H)$. Suppose $j>1$. Since $H\left[\left\{u, v_{1}, u_{1}, v_{j}\right\}\right]$ is not isomorphic to $C_{4}^{\prime}$ or $C_{4}^{\prime \prime}$, we have $v_{j} u_{1}, v_{1} u \in A(H)$. Since $P$ is a shortest path, we have $j=2$ as otherwise $v_{1} u v_{j} u_{j+1} \ldots v_{j} u_{k}$ is shorter than $P$. We have $v_{3} u \notin A(H)$ as otherwise $v_{1} u v_{3} u_{3} \ldots v_{k} u_{k}$ is shorter than $P$. However, $C_{4}^{\prime}$ is isomorphic to $H\left[\left\{v_{2}, u, v_{3}, u_{3}\right\}\right]$, a contradiction.

Now assume that $j=1$. We have $v_{3} u \notin A(H)$ as otherwise we have a shorter path. Since $H$ is semicomplete bipartite, we have $u v_{3} \in A(H)$. However $H\left[\left\{v_{1}, u, v_{3}, u_{2}\right\}\right] \cong C_{4}^{\prime}$, a contradiction. 
Analogously we can prove that $\hat{U} \rightarrow v$ for every $v \in V-\hat{V}$. Consider $u \in U-$ $\hat{U}, v \in V-\hat{V}$. We show that $u v \in A(H)$. Suppose this is not true. We have $v u \in$ $A(H)$. Since $H\left[\left\{v, u, v_{1}, u_{1}\right\}\right]$ is not isomorphic to $\vec{C}_{4}, C_{4}^{\prime}$ or $C_{4}^{\prime \prime}$ we have $v_{1} u, v u_{1} \in A(H)$. Since $H\left[\left\{v, u, v_{k}, u_{k}\right\}\right]$ is not isomorphic to $C_{4}^{\prime}$ or $C_{4}^{\prime \prime}$ we have $v_{k} u, v u_{k} \in A(H)$. But now $\operatorname{dist}\left(v_{1}, u_{k}\right)=3$ in $U N\left(H_{1}\right)$, a contradiction.

Case 2: $d_{1}=4$.

We will show that again $U N\left(H_{2}\right)$ is a complete bipartite graph. Assume that $v_{1} u_{1}^{\prime} v_{2}^{\prime} u_{2}^{\prime} v_{3}^{\prime}$ is a shortest path between a pair $v_{1} \in V$ and $v_{3}^{\prime} \in U$ in $U N\left(H_{1}\right)$. Let $U_{1}=N^{+}\left(v_{1}\right)$, $V_{2}=N^{-}\left(U_{1}\right)-\left\{v_{1}\right\}, U_{2}=N^{+}\left(V_{2}\right)-U_{1}$ and $V_{3}=N^{-}\left(U_{2}\right)-V_{2}$. By the definitions, $V=\left\{v_{1}\right\} \cup V_{2} \cup V_{3}$ and $U=U_{1} \cup U_{2}$. Observe also that $\left(U_{1}, V_{3}\right)=U_{1} \times V_{3},\left(V_{3}, U_{1}\right)=\emptyset$, $\left(U_{2},\left\{v_{1}\right\}\right)=U_{2} \times\left\{v_{1}\right\}$ and $\left(\left\{v_{1}\right\}, U_{2}\right)=\emptyset$. Let $u_{1} \in U_{1}, u_{2} \in U_{2}, v_{2} \in V_{2}$ and $v_{3} \in V_{3}$ be arbitrary. If $u_{2} v_{3} \notin A(H)$, then $v_{3} u_{2} \in A(H)$, and now $H\left[\left\{u_{1}, v_{3}, u_{2}, v_{1}\right\}\right]$ is either $\vec{C}_{4}$ or $C_{4}^{\prime}$, a contradiction. Therefore, we have $u_{2} v_{3} \in A(H)$ and consequently $\left(U_{2}, V_{3}\right)=U_{2} \times V_{3}$. Consider $v_{3}, u_{2}$ where $v_{3} u_{2} \in A(H)$. Since $H\left[\left\{u_{1}, v_{3}, u_{2}, v_{1}\right\}\right]$ is not $C_{4}^{\prime}$ or $\vec{C}_{4}$, we conclude that $u_{1} v_{1} \in A(H)$ and consequently $\left(U_{1},\left\{v_{1}\right\}\right)=U_{1} \times\left\{v_{1}\right\}$.

Note that we have already proved that the underlying graph of $H_{2}\left[U_{1} \cup U_{2} \cup V_{3} \cup\left\{v_{1}\right\}\right]$ is a complete bipartite graph. Suppose $u_{1} v_{2} \in A(H)$. Then $u_{2} v_{2} \in A(H)$ as otherwise $H\left[\left\{u_{1}, v_{1}, u_{2}, v_{2}\right\}\right]$ is isomorphic to $C_{4}^{\prime}$ or $C_{4}^{\prime \prime}$, a contradiction. Therefore, if $u_{1} v_{2} \in A(H)$, then $\left(U_{2},\left\{v_{2}\right\}\right)=U_{2} \times\left\{v_{2}\right\}$. Thus, to show that $\left(U_{2}, V_{2}\right)=U_{2} \times V_{2}$ it suffices to prove that every vertex in $V_{2}$ has an in-neighbor in $U_{1}$. Suppose this is not true, and let $X_{2}$ be the set of all vertices in $V_{2}$ that does not have an in-neighbor in $U_{1}$. Let $Y_{2}$ be the set of all vertices in $U_{2}$ that have an arc into $X_{2}$. As $H$ is strong some vertex in $H$ must have an arc into $X_{2}$, which implies that $Y_{2} \neq \emptyset$.

If there is an arc $v_{3} y_{2}$ from $V_{3}$ to $Y_{2}$, then let $x_{2}$ be an out-neighbor of $y_{2}$ in $X_{2}$ and let $u_{1} \in U_{1}$ be arbitrary. However this is a contradiction to $H\left[\left\{v_{3}, y_{2}, x_{2}, u_{1}\right\}\right]$ not being isomorphic to $C_{4}^{\prime}$ and $C_{4}^{\prime \prime}$, which implies that there is no arc from $V_{3}$ to $Y_{2}$.

As $H$ is strong and there is no arc from $V_{3}$ into $U_{1}$ or $Y_{2}$, there must be an arc, say $v_{3} u_{2}$, from $V_{3}$ into $U_{2}-Y_{2}$. As $H$ is strong there must also be an arc from $V(H)-X_{2}-Y_{2}$ into $X_{2} \cup Y_{2}$. By the above this arc, say $v_{2} y_{2}$, must be from $V_{2}-X_{2}$ to $Y_{2}$. As $y_{2}$ belongs to $Y_{2}$ there must be a vertex, say $x_{2} \in X_{2}$, such that $y_{2} x_{2} \in A(H)$. As $v_{2} \notin X_{2}$ we note that there is a vertex, say $u_{1} \in U_{1}$, such that $u_{1} v_{2} \in A(H)$. As $u_{1} x_{2} \notin A(H)$ and $H\left[\left\{v_{2}, x_{2}, u_{1}, y_{2}\right\}\right]$ is not isomorphic to $C_{4}^{\prime}$ and $C_{4}^{\prime \prime}$, we note that $x_{2} y_{2}, v_{2} u_{1} \in A(H)$. if $u_{2} v_{2} \notin A(H)$, then $H\left[\left\{v_{1}, v_{2}, u_{1}, u_{2}\right\}\right]$ is isomorphic to $C_{4}^{\prime}$ and $C_{4}^{\prime \prime}$ (as $v_{1} u_{2} \notin A(H)$ ), a contradiction. As $u_{2} v_{2} \in$ $A(H)$ and $H\left[\left\{v_{2}, v_{3}, u_{2}, y_{2}\right\}\right]$ is not isomorphic to $C_{4}^{\prime}$ and $C_{4}^{\prime \prime}$ we note that $y_{2} v_{2} \in A(H)$ (as $\left.v_{3} y_{2} \notin A(H)\right)$. As $H\left[\left\{v_{2}, x_{2}, u_{2}, y_{2}\right\}\right]$ is not isomorphic to $C_{4}^{\prime}$ and $C_{4}^{\prime \prime}$ we note that $v_{2} u_{2} \in$ $A(H)$ (as $u_{2} x_{2} \notin A(H)$ ). However, the underlying graph of $H\left[v_{1}, u_{1}, v_{2}, v_{3}, u_{2}^{\prime \prime}, v_{2}^{\prime \prime}, u_{2}\right]^{\leftrightarrow}$ is now a bipartite claw, with edges $\left\{v_{2} u_{1}, v_{2} y_{2}, v_{2} u_{2}, u_{1} v_{1}, y_{2} x_{2}, u_{2} v_{3}\right\}$, a contradiction. Therefore $U_{2} \rightarrow V_{2}$. 
We show that $u_{1} v_{2} \in A(H)$ for every $u_{1} \in U_{1}$ and $v_{2} \in V_{2}$. Suppose this is not true for some $v_{2} \in V_{2}$ and $u_{1} \in U_{1}$. Then we have $v_{2} u_{1} \in A(H)$. Let $v_{3} \in V_{3}$ be arbitrary and $u_{2} \in U_{2} \cap N^{+}\left(v_{3}\right)$. Then $H\left[\left\{u_{1}, v_{3}, u_{2}, v_{2}\right\}\right]$ is isomorphic to $C_{4}^{\prime}$ or $C_{4}^{\prime \prime}$. This completes our proof that $U N\left(H_{2}\right)$ is a complete bipartite graph.

Case 3: $d_{1}=3$.

Consider a Min-Max ordering $\pi$ for $H_{1}$. Let $\pi(x)=\min \left\{\pi\left(x^{\prime}\right): x^{\prime} \in V\right\}, \pi(t)=$ $\max \left\{\pi\left(t^{\prime}\right): t^{\prime} \in U\right\}, \pi(y)=\max \left\{\pi\left(y^{\prime}\right): y^{\prime} \in U, x \rightarrow y^{\prime}\right\}$, and $\pi(z)=\min \left\{\pi\left(z^{\prime}\right): z^{\prime} \in\right.$ $\left.V, z^{\prime} \rightarrow t\right\}$.

Let $T=N^{-}(t)$. Since $H$ is strong, every vertex of $V$ has an out-neighbor. Since $\pi$ is a Min-Max ordering, $z^{\prime} \rightarrow t$ for each $z^{\prime} \in V$ with $\pi\left(z^{\prime}\right) \geq \pi(z)$. Thus, $T=\left\{z^{\prime} \in\right.$ $\left.V: \pi\left(z^{\prime}\right) \geq \pi(z)\right\}$. Let $X=N^{+}(x)$. Since $H$ is strong, every vertex of $U$ has an inneighbor. Since $\pi$ is a Min-Max ordering, $x \rightarrow y^{\prime}$ for each $y^{\prime} \in U$ with $\pi\left(y^{\prime}\right) \leq \pi(y)$. Thus, $X=\left\{y^{\prime} \in U: \pi\left(y^{\prime}\right) \leq \pi(y)\right\}$.

Since $\operatorname{dist}(x, z)=2$ in $U N\left(H_{1}\right)$, we have $z \rightarrow y^{\prime}$ for some $y^{\prime} \in N^{+}(x)$. Since $\pi$ is a Min-Max ordering, $z \rightarrow y$ (consider the arcs $z y^{\prime}$ and $x y$ ). Now for every $z^{\prime \prime} \in V$ with $\pi\left(z^{\prime \prime}\right) \leq \pi(z)$, we have $z^{\prime \prime} \rightarrow y$ (as $\pi$ is a Min-Max ordering). Similarly, for every $y^{\prime \prime} \in U$ with $\pi\left(y^{\prime \prime}\right) \geq \pi(y)$, we have $z \rightarrow y^{\prime \prime}$.

Let $\pi(w)=\min \left\{\pi\left(w^{\prime}\right): w^{\prime} \in U\right\}$. Notice that $\operatorname{dist}(w, t)=2$ in $U N\left(H_{1}\right)$. Thus, for some $z^{\prime} \in T$, we have $z^{\prime} \rightarrow w$. Hence, $z \rightarrow w$ and $z \rightarrow X$ (as $\pi$ is a Min-Max ordering). We conclude that $z \rightarrow U$. Similarly, we can obtain that $V \rightarrow y$.

Let $Y=V-T$ and $Z=U-X$. Let $x^{\prime} \in X, y^{\prime} \in Y$. We have $z \rightarrow x^{\prime}$ and $y^{\prime} \rightarrow y$. Hence (as $\pi$ is a Min-Max ordering), $y^{\prime} \rightarrow x^{\prime}$. Thus, $Y \rightarrow X$. Analogously, we can prove that $T \rightarrow Z$.

Let $x^{\prime} \in X, t^{\prime} \in T, y^{\prime} \in Y$ and $x^{\prime} \rightarrow t^{\prime}$. Then $t^{\prime} t, t y^{\prime}, y^{\prime} x^{\prime} \in A(H)$. Since $H\left[\left\{x^{\prime}, t, t^{\prime}, y^{\prime}\right\}\right]$ is not isomorphic to $\vec{C}_{4}, C_{4}^{\prime}$ or $C_{4}^{\prime \prime}$, we have $x^{\prime} \rightarrow y^{\prime}$ and $t \rightarrow t^{\prime}$. Thus, if $x^{\prime} \rightarrow t^{\prime}$, we have $x^{\prime} \rightarrow Y$. Analogously, if $x^{\prime} \rightarrow t^{\prime}$, we have $Z \rightarrow t^{\prime}$. Hence,

$$
x^{\prime} \rightarrow t^{\prime} \text { implies } x^{\prime} \rightarrow Y \text { and } Z \rightarrow t^{\prime}
$$

We will now prove the following: for a pair $u, u^{\prime}$ of distinct vertices of $U$ we have $N^{+}(u) \subseteq N^{+}\left(u^{\prime}\right)$ or $N^{+}\left(u^{\prime}\right) \subseteq N^{+}(u)$. By Lemma 3.1 this implies that $H$ has a 2Min-Max ordering and we are done. Suppose that we have neither $N^{+}(u) \subseteq N^{+}\left(u^{\prime}\right)$ nor $N^{+}\left(u^{\prime}\right) \subseteq N^{+}(u)$. Thus, there is a pair $v, v^{\prime}$ of vertices in $V$ such that $u \rightarrow v, u^{\prime} \rightarrow v^{\prime}$, but $u v^{\prime}$ and $u^{\prime} v$ are not arcs in $H$. Since $H\left[\left\{u, u^{\prime}, v, v^{\prime}\right\}\right]$ is not isomorphic to $\vec{C}_{4}, C_{4}^{\prime}$ and $C_{4}^{\prime \prime}$, we have $v \rightarrow u$ and $v^{\prime} \rightarrow u^{\prime}$. Now consider four cases.

Case 3.1: $v, v^{\prime} \in Y$. Let $t^{\prime} \in T$. By the definition of $t, t \notin\left\{u, u^{\prime}\right\}$. If $u^{\prime} \rightarrow t^{\prime}$, then $H\left[\left\{t, t^{\prime}, v, u^{\prime}\right\}\right]$ is isomorphic to $\vec{C}_{4}, C_{4}^{\prime}$ or $C_{4}^{\prime \prime}$, which is impossible. Thus, $u^{\prime} t^{\prime} \notin A(H)$ and $t^{\prime} \rightarrow u^{\prime}$. Analogously, $u t^{\prime} \notin A(H)$ and $t^{\prime} \rightarrow u$. By the fact that $t^{\prime} \rightarrow t$ and the existence and nonexistence of previously considered arcs, we conclude that $H\left[\left\{v, v^{\prime}, u, u^{\prime}, t, t^{\prime}\right\}\right]$ is 
isomorphic to $N_{1}$ or $N_{2}$, which is impossible.

Case 3.2: $u, u^{\prime} \in Z$. We can show that this case is impossible similarly to Case 3.1 but considering $x, w$ instead of $t, t^{\prime}$.

Case 3.3: $v \in Y, v^{\prime} \in T$. By (3),$u^{\prime} \in Z$. By Case 3.2, we may assume that $u \in X$. Then $H\left[\left\{v, v^{\prime}, u^{\prime}, t\right\}\right]$ is isomorphic to $\vec{C}_{4}, C_{4}^{\prime}$ or $C_{4}^{\prime \prime}$, which is impossible.

Case 3.4: $v, v^{\prime} \in T$. By Case 3.2, we may assume that $u \in X$. By (3), $Z \rightarrow v$ and, thus, $u^{\prime} \in X$. By (3), we conclude that $u x u, u^{\prime} x u^{\prime}$, vtv and $v^{\prime} t v^{\prime}$ are 2-cycles. Notice that $t \rightarrow x$, but $x t \notin A(H)$. Now it follows that $H\left[\left\{x, v, v^{\prime}, u, u^{\prime}, t\right\}\right]^{\leftrightarrow}$ is isomorphic to $C_{6}$, a contradiction.

It follows from Cases 1,2 and 3 that if neither $U N\left(H_{1}\right)$ nor $U N\left(H_{2}\right)$ are complete bipartite graphs, then we must have $d_{1}=d_{2}=3$. In this case we have shown that for every pair $u, u^{\prime}$ of distinct vertices of $U$ we have $N^{+}(u) \subseteq N^{+}\left(u^{\prime}\right)$ or $N^{+}\left(u^{\prime}\right) \subseteq N^{+}(u)$. However, by swapping the roles of $U$ and $V$ we also get that for every pair $v, v^{\prime}$ of distinct vertices of $V$ we have $N^{+}(v) \subseteq N^{+}\left(v^{\prime}\right)$ or $N^{+}\left(v^{\prime}\right) \subseteq N^{+}(v)$.

The following theorem shows when $\operatorname{MinHOM}(H)$ is polynomial time solvable for the case when $H$ is not strong, and does not contain $\vec{C}_{4}$ as an induced subdigraph.

Theorem 3.3 Let $H=(U, V ; A)$ be a semicomplete bipartite digraph with strong components $C_{1}, C_{2}, \ldots, C_{p}(p \geq 2)$ satisfying the following:

- There is no arc from $C_{i}$ to $C_{j}$ for $i>j$,

- $H$ does not contain an induced subdigraph belonging to $\mathcal{H} \mathcal{F O R B}$ or an induced directed 4-cycle.

Then $H$ has a 2-Min-Max ordering and MinHOM(H) is polynomial time solvable.

Proof: Suppose there are $v, v^{\prime} \in V$ and $u, u^{\prime} \in U$ such that $A\left(H^{\rightarrow}\left[\left\{v, v^{\prime}, u, u^{\prime}\right\}\right]\right)=$ $\left\{v u, v^{\prime} u^{\prime}\right\}$. Note that $\left\{v, v^{\prime}, u, u^{\prime}\right\}$ belong to a strong component of $H$ as they are contained in a 4-cycle. Let $\left\{v, v^{\prime}, u, u^{\prime}\right\} \subseteq V\left(C_{t}\right)$ for some $t$ and let $V_{1}=\left\{x \in V \mid x \in C_{i}, i<t\right\}$, $U_{1}=\left\{y \in U \mid y \in C_{i}, i<t\right\}, V_{2}=V \cap C_{t}, U_{2}=U \cap C_{t}, V_{3}=V-V_{1}-V_{2}$ and $U_{3}=U-U_{1}-U_{2}$.

If there is a $w^{\prime} \in U_{3}$ and $w \in V_{3}$, such that $w^{\prime} \rightarrow w$, then $H\left[\left\{u, v, u^{\prime}, v^{\prime}, w, w^{\prime}\right\}\right]$ is either the dual of $N_{1}$ or $N_{2}$. Therefore we must have $A\left(H\left[U_{3} \cup V_{3}\right]\right)=V_{3} \times U_{3}$. Analogously we must have $A\left(H\left[U_{1} \cup V_{1}\right]\right)=V_{1} \times U_{1}$. Consider $H^{\prime}=H\left[U_{2} \cup V_{2}\right]$ and note that $H^{\prime}$ is strong and does not contain a digraph from $\mathcal{H} \mathcal{F O R \mathcal { B }}$ or $\vec{C}_{4}$ as an induced subdigraph. Therefore Theorem 3.2 implies that $U_{2} \rightarrow V_{2}$ (as $V_{2} \rightarrow U_{2}$ is not true), which furthermore implies that $\left(U_{1} \cup U_{2}\right) \rightarrow\left(V_{2} \cup V_{3}\right)$. 
Let $\pi$ be a min-max ordering of $H^{\rightarrow}$. Let $u v \in A(H)$ and $u^{\prime} v^{\prime} \in A(H)$ be two distinct arcs from $U$ to $V$. As $d^{+}(u)>0$ we note that $u \in U_{1} \cup U_{2}$, by the above. Analogously we note that $u^{\prime} \in U_{1} \cup U_{2}, v \in V_{2} \cup V_{3}$ (as $d^{-}(v)>0$ ) and $v^{\prime} \in V_{2} \cup V_{3}$. By the above we therefore have $u v^{\prime}, u^{\prime} v \in A(H)$. As $u, u^{\prime}, v, v^{\prime}$ were chosen arbitrarily, this implies that $\pi$ is a 2-Min-Max ordering.

If there are no $v, v^{\prime} \in V$ and $u, u^{\prime} \in U$ such that $A\left(H^{\rightarrow}\left[\left\{v, v^{\prime}, u, u^{\prime}\right\}\right]\right)=\left\{v u, v^{\prime} u^{\prime}\right\}$. Then $H \rightarrow$ satisfies the condition of Lemma 3.1. Therefore $H$ has the 2-Min-Max ordering. $\diamond$

The following theorem shows when $\operatorname{MinHOM}(H)$ is polynomial time solvable for the case when $H$ does contain $\vec{C}_{4}$ as an induced subdigraph.

Theorem 3.4 Let $H$ be a semicomplete bipartite digraph. Assume that $H$ does not contain a digraph from $\mathcal{H} \mathcal{F O R \mathcal { B }}$ as an induced subdigraph, but contains $\vec{C}_{4}$ as an induced subdigraph. Then $H$ is an extension of a $\vec{C}_{4}$ and $\operatorname{MinHOM}(H)$ is polynomial time solvable.

Proof: Observe that an extension $L$ of any cycle $\vec{C}_{p}, p \geq 2$, has a $p$-Min-Max ordering. Thus, $\operatorname{MinHOM}(L)$ is polynomial time solvable by Theorem 2.2. Let $C=v_{1} u_{1} v_{2} u_{2} v_{1}$ be an induced 4-cycle of $H$. It suffices to prove that $H$ is an extension of $C$.

For $i=1,2$, let $M^{+}\left(v_{i}\right)=\left\{u \in U: v_{i} u \in A(H), u v_{i} \notin A(H)\right\}, M^{-}\left(v_{i}\right)=\{u \in$ $\left.U: u v_{i} \in A(H) \quad v_{i} u \notin A(H)\right\}$, and $M\left(v_{i}\right)=\left\{u \in U: u v_{i}, v_{i} u \in A(H)\right\}$. We have $M^{+}\left(v_{1}\right) \cap M^{+}\left(v_{2}\right)=\emptyset$ as otherwise $H\left[\left\{v_{1}, v_{2}, u_{1}, u_{2}, u_{3}\right\}\right] \cong H^{*}$, where $u_{3} \in N^{+}\left(v_{1}\right) \cap$ $N^{+}\left(v_{2}\right)$. We have $M^{-}\left(v_{1}\right) \cap M^{-}\left(v_{2}\right)=\emptyset$ as otherwise $H\left[\left\{v_{1}, v_{2}, u_{1}, u_{2}, u_{3}\right\}\right] \cong H^{* *}$, where $u_{3} \in N^{-}\left(v_{1}\right) \cap N^{-}\left(v_{2}\right)$ and $H^{* *}$ is the converse of $H^{*}$.

We have $M\left(v_{1}\right) \cap\left(M^{+}\left(v_{2}\right) \cup M^{-}\left(v_{2}\right)\right) \neq \emptyset$ as otherwise $H\left[\left\{v_{1}, u_{1}, v_{2}, u\right\}\right] \cong C_{4}^{\prime}$, where $u \in M\left(v_{1}\right) \cap M^{+}\left(v_{2}\right)$ or $H\left[\left\{v_{1}, u_{2}, v_{2}, u\right\}\right] \cong C_{4}^{\prime}$, where $u \in M\left(v_{1}\right) \cap M^{-}\left(v_{2}\right)$. Moreover, $M\left(v_{1}\right) \cap M\left(v_{2}\right)=\emptyset$ as otherwise $H\left[\left\{v_{1}, u_{1}, v_{2}, u\right\}\right] \cong C_{4}^{\prime \prime}$, where $u \in M\left(v_{1}\right) \cap M\left(v_{2}\right)$. The arguments above imply that $M\left(v_{1}\right)=M\left(v_{2}\right)=\emptyset$, and $M^{+}\left(v_{1}\right)=M^{-}\left(v_{2}\right)$ and $M^{-}\left(v_{1}\right)=M^{+}\left(v_{2}\right)$.

Similarly, we can define $M+\left(u_{i}\right), M^{-}\left(u_{i}\right)$ and $M\left(u_{i}\right), i=1,2$, and prove the relations analogous to those for $M^{+}\left(v_{i}\right), M^{-}\left(v_{i}\right)$ and $M\left(v_{i}\right), i=1,2$.

Let $v \in V-\left\{v_{1}, v_{2}\right\}$ and $u \in U-\left\{u_{1}, u_{2}\right\}$ be arbitrary. Without loss of generality, assume that $u \in M^{+}\left(v_{1}\right)=M^{-}\left(v_{2}\right)$ and $v \in M^{+}\left(u_{2}\right)=M^{-}\left(u_{1}\right)$ (all other cases can be treated similarly). To show that $H$ is an extension of $C$, it suffices to prove that $v \rightarrow u$, but $u v \notin A(H)$. Suppose first that $u \rightarrow v$ and $v \rightarrow u$. Then $H\left[\left\{v, u, v_{2}, u_{2}\right\}\right] \cong C_{4}^{\prime}$, a contradiction. Now suppose that $u \rightarrow v$, but $v u \notin A(H)$. Then $H\left[\left\{v, v_{1}, v_{2}, u, u_{2}\right\}\right] \cong H^{*}$, a contradiction. Thus, $v \rightarrow u$, but $u v \notin A(H)$ and we are done. 
The three theorems of this section and the fact that $\vec{C}_{4}$ has a 4-Min-Max ordering imply the following:

Corollary 3.5 Let $H$ be a connected semicomplete bipartite digraph not containing a digraph from $\mathcal{H} \mathcal{F O R B}$ as an induced subdigraph. Then $\operatorname{MinHOM}(H)$ is polynomial time solvable and $H$ has a $k$-Min-Max ordering for $k=2$ or 4 .

\section{NP-hardness Cases}

It is well known that the problem of finding a maximum size independent set in an undirected graph $G$ is NP-hard. We say that a set $I$ in a digraph $D$ is independent if no vertices in $I$ are adjacent. Clearly, the problem of finding a maximum size independent set in a digraph $D$ (MISD) is NP-hard.

Lemma 4.1 $\operatorname{MinHOM}\left(C_{4}^{\prime}\right)$ is NP-hard.

Proof: Let $H$ be isomorphic to $C_{4}^{\prime}$ as follows: $V(H)=\{1,2,3,4\}$ and $A(H)=\{12,21,23,34,41\}$. Let $D$ be an arbitrary digraph. We replace every arc $u v$ of $D$ by the digraph $G_{u v}$ with $V\left(G_{u v}\right)=\{x, y, z, u, v\}$ and $A\left(G_{u v}\right)=\{u x, x y, y z, v z\}$. Let $D^{\prime}$ be the obtained digraph. Define the cost function as follows: $c_{1}(u)=1, c_{2}(u)=1, c_{3}(u)=0, c_{4}(u)=1, c_{1}(v)=1$, $c_{2}(v)=1, c_{3}(v)=0, c_{4}(v)=1, c_{i}(t)=0$ when $t \notin\{u, v\}$ and $i \in\{1,2,3,4\}$.

Let $h$ be a mapping from $V\left(G_{u v}\right)$ to $V(H)$, and let $u v$ be an arc in $D$. If $h(u)=$ $h(y)=h(v)=1$ and $h(x)=h(z)=2$, then $h$ is a homomorphism. Thus, there is a homomorphism of $D^{\prime}$ to $H$, which maps all vertices of $D$ into 1 and the vertices of $D^{\prime}$ not in $D$ into 1 or 2 .

Now let $f$ be a homomorphism of $G_{u v}$ to $H$. Observe that if $f(u)=1$, then $f(x)=2$, $f(y)$ is either 1 or $3, f(z)$ is either 2 or $4, f(v)$ is either 1 or 3 . . Similarly if $f(u)=3$, then $f(v)=1$; if $f(u)=4$, then $f(v)$ is either 2 or 4 ; if $f(u)=2$, then $f(v)$ is either 2 or 4 .

Let $g$ be a minimum cost homomorphism of $D^{\prime}$ to $H$ and let $S=\{s \in V(D): g(s)=$ $3\}$. Notice that the cost of $g$ is $|V(D)|-|S|$ and $S$ is an independent set by the arguments of the previous paragraph. Thus, $S$ is an independent set of $D$ of maximum size.

Let $I$ be a maximum size independent set in $D$. The above arguments show that there is a homomorphism $d$ of $D^{\prime}$ to $H$ such that $d(t)=3$ if $t \in I$ and $d(t)=1$ if $t \in V(D)-I$. Notice that $d$ is a minimum cost homomorphism.

Now we can conclude that $\operatorname{MinHOM}(H)$ is NP-hard since MISD is NP-hard. $\diamond$

Lemma 4.2 $\operatorname{MinHOM}\left(C_{4}^{\prime \prime}\right)$ is NP-hard. 
Proof: Let $H$ be isomorphic to $C_{4}^{\prime \prime}$ as follows: $V(H)=\{1,2,3,4\}, A(H)=\{12,21,23,32,34,41\}$. Let $D$ be an arbitrary digraph. We replace every arc $u v$ of $D$ by the digraph $G_{u v}$ with $V\left(G_{u v}\right)=\{x, u, v\}$ and $A\left(G_{u v}\right)=\{u x, x v\}$. Let $D^{\prime}$ be the obtained digraph. Define the cost function as follows: $c_{1}(u)=1, c_{2}(u)=1, c_{3}(u)=1, c_{4}(u)=0, c_{1}(v)=1, c_{2}(v)=1$, $c_{3}(v)=1, c_{4}(v)=0, c_{i}(x)=0$ for $i=1,2,3,4$.

Let $h$ be a mapping from $V\left(G_{u v}\right)$ to $V(H)$, and let $u v$ be an arc in $D$. If $h(u)=h(v)=1$ and $h(x)=2$, then $h$ is a homomorphism. Thus, there is a homomorphism of $D^{\prime}$ to $H$, which maps all vertices of $D$ into 1 and the vertices of $D^{\prime}$ not in $D$ into 2 .

Now let $f$ be a homomorphism of $G_{u v}$ to $H$. Observe that if $f(u)=1$, then $f(x)=2$ and $f(v)$ is either 1 or 3. Similarly if $f(u)=2$, then $f(v) \in\{2,4\}$; if $f(u)=3$, then $f(v) \in\{1,3\}$; if $f(u)=4$, then $f(v)=2$.

Let $g$ be a minimum cost homomorphism of $D^{\prime}$ to $H$ and let $S=\{s \in V(D): g(s)=$ 4 . Notice that the cost of $g$ is $|V(D)|-|S|$ and $S$ is an independent set by the arguments of the previous paragraph. Thus, $S$ is an independent set of $D$ of maximum size.

The rest of the proof is similar to that of Lemma 4.1 .

The following lemma was stated in [5]. We give a proof here for the sake of completeness.

Lemma 4.3 $\operatorname{MinHOM}\left(H^{*}\right)$ is NP-hard.

Proof: Let $H$ be isomorphic to $H^{*}$ as follows: $V(H)=\{1,2,3,4,5\}, A(H)=\{12,23,34,41,15,35\}$. We replace every arc $u v$ of $D$ by the digraph $G_{u v}$ with $V\left(G_{u v}\right)=\left\{v_{1}, v_{2}, v_{3}, v_{4}, v_{5}, v_{6}, v_{7}\right\}$, where $u=v_{6}$ and $v=v_{7}$, and $A\left(G_{u v}\right)=\left\{v_{1} v_{2}, v_{2} v_{3}, v_{3} v_{4}, v_{4} v_{1}, v_{5} v_{6}, v_{5} v_{7}, v_{1} v_{6}, v_{3} v_{7}\right\}$. Let $D^{\prime}$ be the obtained digraph. Define the cost function as follows: $c_{1}\left(v_{6}\right)=1, c_{2}\left(v_{6}\right)=1$, $c_{3}\left(v_{6}\right)=1, c_{4}\left(v_{6}\right)=0, c_{5}\left(v_{6}\right)=1, c_{1}\left(v_{7}\right)=1, c_{2}\left(v_{7}\right)=1, c_{3}\left(v_{7}\right)=1, c_{4}\left(v_{7}\right)=0$, $c_{5}\left(v_{7}\right)=1$, and $c_{i}\left(v_{j}\right)=0$ for each $j \neq 6,7$.

Let $h$ be a mapping from $V\left(G_{u v}\right)$ to $V(H)$, and let $u v$ be an arc in $D$. If $h\left(v_{i}\right)=i$ for each $i=1,2,3,4, h\left(v_{5}\right)=1$ and $h(u)=h(v)=5$, then $h$ is a homomorphism. Thus, there is a homomorphism of $D^{\prime}$ to $H$, which maps all vertices of $D$ into 5 .

Now let $f$ be a homomorphism of $G_{u v}$ to $H$. Observe that if $x=f(u)=f(v)$, then $x=5$. Also, if $f(u)=5$ then $f(v) \in\{2,4,5\}$ and if $f(v)=5$ then $f(u) \in\{2,4,5\}$.

Let $g$ be a minimum cost homomorphism of $D^{\prime}$ to $H$ and let $S=\{s \in V(D): g(s)=$ $4\}$. Notice that the cost of $g$ is $|V(D)|-|S|$ and $S$ is an independent set by the arguments of the previous paragraph. Thus, $S$ is an independent set of $D$ of maximum size.

The rest of the proof is similar to that of Lemma 4.1

Lemma 4.4 $\operatorname{MinHOM}\left(N_{1}\right)$ is NP-hard. 
Proof: We shall reduce the maximum independent set problem to $\operatorname{MinHOM}\left(N_{1}\right)$. Let $H$ be the following digraph isomorphic to $N_{1}: V(H)=\{1,2,3,4,5,6\}$,

$$
A(H)=\{12,21,34,43,56,65,23,25,14,16,54,36\} .
$$

Let $D$ be an arbitrary digraph. We replace every arc $u v$ of $D$ with the digraph $G_{u v}$ with $V\left(G_{u v}\right)=\{x, y, z, u, v\}$ and $A\left(G_{u v}\right)=\{u x, v z, x y, y z\}$. Consider the following cost function: $c_{2}(u)=c_{2}(v)=1, c_{6}(u)=c_{6}(v)=0, c_{i}(u)=c_{i}(v)=2 M+1$ for $i \neq 2,6$ and $c_{6}(y)=2 M+1$, where $M=|V(D)|$. In all remaining cases the cost is zero. Let $D^{\prime}$ be the obtained digraph, let $f$ be a mapping from $V\left(D^{\prime}\right)$ to $V(H)$, and let $u v$ be an arc in $D$.

Assume that $f(u)=f(v)=2$. Then with $f(x)=f(z)=1$ and $f(y)=2$, we obtain a homomorphism from $G_{u v}$ to $H$ of cost 2. This implies there is a homomorphism of $D^{\prime}$ to $H$ of cost $2 M<2 M+1$, and, thus, every vertex of $D$ in $D^{\prime}$ must be colored either 2 or 6 in any minimum cost homomorphism of $D^{\prime}$ to $H$. Let $f$ be a homomorphism of $D^{\prime}$ to $H$ and let us consider the remaining options for coloring the vertices of $D$ in $D^{\prime}$.

Assume that $f(v)=6$ and $f(u)=2$. Then with $f(z)=5, f(y)=2$ and $f(x)=1$, we obtain a homomorphism from $G_{u v}$ to $H$ of cost 1 . Assume that $f(v)=2$ and $f(u)=6$. Then with $f(x)=5, f(y)=4$ and $f(z)=3$, we obtain a homomorphism from $G_{u v}$ to $H$ of cost 1. Note that if $f(u)=f(v)=6$, then $f(x)=f(z)=5$ and $f(y)=6$. Then the cost of $f$ will be at least $2 M+1$ implying we cannot color both vertices $u$ and $v$ in color 6 in any minimum cost homomorphism of $D^{\prime}$ to $H$.

Now let $f$ be a minimum cost homomorphism, let $S$ be the vertices of $D$ in $D^{\prime}$ colored 6 and $T=V(D)-S$. Recall that the vertices of $T$ are colored 2. Notice that $S$ is an independent set and the cost of $f$ equals $|T|$.

The rest of the proof is similar to that of Lemma 4.1

Lemma 4.5 MinHOM(N $\left(N_{2}\right)$ is NP-hard.

We shall reduce the maximum independent set problem to $\operatorname{MinHOM}\left(N_{2}\right)$. Let $H$ be the following digraph isomorphic to $N_{2}: V(H)=\{1,2,3,4,5,6\}$,

$$
A(H)=\{12,34,43,56,65,23,25,14,16,54,36\} .
$$

Let $D$ be an arbitrary digraph. We replace every arc $u v$ of $D$ by the digraph $G_{u v}$ with $V\left(G_{u v}\right)=\{x, y, z, u, v\}$ and $A\left(G_{u v}\right)=\{u x, v z, x y, z y\}$. We introduce the following cost function: $c_{1}(u)=c_{1}(v)=1, c_{5}(u)=c_{5}(v)=0, c_{i}(u)=c_{i}(v)=2 M+1$ for $i \neq 1,5$ and $c_{4}(x)=2 M+1$ and $c_{6}(z)=2 M+1$, where $M=|V(D)|$. In any other cases the cost is zero. Let $D^{\prime}$ be the obtained digraph, let $f$ be a mapping from $V\left(D^{\prime}\right)$ to $V(H)$, and let $u v$ be an $\operatorname{arc}$ in $D$.

Assume that $f(u)=f(v)=1$. With $f(x)=f(z)=2$ and $f(y)=3$ with obtain a homomorphism from $H^{\prime}$ to $H$ with cost 2. Thus, there is a homomorphism of $D^{\prime}$ to $H$ of 
cost at most $2 M$ (assign all vertices of $D$ in $D^{\prime}$ color 2) and no vertex of $D$ in $D^{\prime}$ must not be assigned any color other than 1 and 5. Let $f$ be a homomorphism of $D^{\prime}$ to $H$ and let us consider the remaining options for coloring the vertices of $D$ in $D^{\prime}$.

Assume that $f(v)=1$ and $f(u)=5$. With $f(z)=2, f(x)=6$ and $f(y)=5$, we obtain a homomorphism of $G_{u v}$ to $H$ of cost 1 . Assume that $f(v)=5$ and $f(u)=1$. With $f(x)=2, f(z)=4$ and $f(y)=3$, we obtain a homomorphism of $G_{u v}$ to $H$ of cost 1. Note that if $f(u)=f(v)=5$, then $f(x) \in\{4,6\}$ and $f(z) \in\{4,6\}$. Thus, $f$ has cost at least $2 M+1$ implying that a minimum cost homomorphism of $D^{\prime}$ to $H$ does not assign adjacent vertices of $D$ color 5 (in $D^{\prime}$ ).

Now let $f$ be a minimum cost homomorphism, let $S$ be the vertices of $D$ in $D^{\prime}$ colored 5 and $T=V(D)-S$. Recall that the vertices of $T$ are colored 1 . Notice that $S$ is an independent set and the cost of $f$ equals $|T|$.

The rest of the proof is similar to that of Lemma 4.1.

Corollary 4.6 $\operatorname{MinHOM}(H)$ is $N P$-hard for every $H \in \mathcal{H} \mathcal{F O} \mathcal{R B}$.

Proof: If $H$ is isomorphic to $C_{4}^{\prime}, C_{4}^{\prime \prime}, H^{*}, N_{1}$ or $N_{2}$ or the converse of one of the five digraphs, then $\operatorname{MinHOM}(H)$ is NP-hard due to the lemmas of this section and the simple fact that if $\operatorname{MinHOM}(H)$ is NP-hard and $H^{\prime}$ is the converse of $H$ then $\operatorname{MinHOM}\left(H^{\prime}\right)$ is NP-hard as well.

Let $\mathcal{B}$ be the set consisting of the following bipartite graphs: bipartite claw, bipartite net, bipartite tent and every even cycle with at least 6 vertices. If $U N\left(H^{s}\right)$, where $s \in$ $\{\rightarrow, \leftarrow\}$, is isomorphic to a graph in $\mathcal{B}$, then $\operatorname{MinHOM}(H)$ is NP-hard due to Theorem 1.2 and the transformation from a bipartite undirected graph to a semicomplete bipartite digraph described in the last paragraph of subsection 'Minimum Cost Homomorphisms' of Section 1. If $U N\left(H^{\leftrightarrow}\right)$ is isomorphic to a graph in $\mathcal{B}$, then $\operatorname{MinHOM}(H)$ is NP-hard as, for each bipartite undirected graph $L, \operatorname{MinHOM}(L)$ is equivalent to $\operatorname{MinHOM}\left(L^{+}\right)$, where $L^{+}$is the digraph obtained from $L$ by replacing every edge $x y$ with two arcs $x y$ and $y x . \diamond$

\section{Dichotomy for semicomplete multipartite digraphs}

A digraph $D$ is called semicomplete $k$-partite if $D$ can be obtained from a complete $k$ partite (undirected) graph $G$ by replacing every edge $x y$ of $G$ by either the arc $x y$ or the arc $y x$ or the pair $x y, y x$ of arcs. Let $T T_{p}$ denote the acyclic tournament on $p \geq 1$ vertices. Let $p \geq 3$ and let $T T_{p}^{-}$be a digraph obtained from $T T_{p}$ by deleting the arc from the vertex of in-degree zero to the vertex of out-degree zero. Combining the main result of this paper with the main result of [5], we obtain the following:

Theorem 5.1 Let $H$ be a semicomplete $k$-partite digraph. If $k=2$ and $H$ does not 
contain a digraph from $\mathcal{H} \mathcal{F O R B}$ as an induced subdigraph or if $k \geq 3$ and $H$ is an extension of either $T T_{k}$ or $T T_{k+1}^{-}$or $\vec{C}_{3}$, then $\operatorname{MinHOM}(H)$ is polynomial time solvable. Otherwise, $\operatorname{MinHOM}(H)$ is NP-hard.

\section{Further Research}

In the case of undirected graphs $H$, the well-known theorem of Hell and Nešetřil [9] on the homomorphism problem implies that $\operatorname{MinHOM}(H)$ is NP-hard for each non-bipartite graph $H$. The authors of [3] obtained a complete dichotomy of the computational complexity of $\operatorname{MinHOM}(H)$ when $H$ is undirected. The dichotomy obtained in this paper significantly extends the dichotomy of [3]. This indicates that the problem of obtaining a dichotomy for the computational complexity of $\operatorname{MinHOM}(H)$ when $H$ is a bipartite digraph is a very difficult problem. Note that $\operatorname{MinHOM}(H)$ is polynomial-time solvable for some non-bipartite digraphs, for example, for acyclic tournaments [5]. Thus, a dichotomy for bipartite directed case does not coincide with a dichotomy for the general directed case. The problem of obtaining dichotomy for both cases is a very interesting open problem.

Acknowledgements We are grateful to Pavol Hell for several useful discussions. Research of the first author was supported in part by the IST Programme of the European Community, under the PASCAL Network of Excellence, IST-2002-506778.

\section{References}

[1] J. Bang-Jensen and G. Gutin, Digraphs: Theory, Algorithms and Applications, SpringerVerlag, London, 2000.

[2] D. Cohen, M. Cooper, P. Jeavons, and A. Krokhin, A maximal tractable class of soft constraints. J. Artif. Intell. Res. 22 (2004), 1-22.

[3] G. Gutin, P. Hell, A. Rafiey and A. Yeo, Minimum Cost Homomorphisms to Proper Interval Graphs and Bigraphs. Submitted to Europ. J. Combin.

[4] G. Gutin, A. Rafiey and A. Yeo, Minimum Cost and List Homomorphisms to Semicomplete Digraphs. Discrete Appl. Math. 154 (2006), 890-897.

[5] G. Gutin, A. Rafiey and A. Yeo, Minimum Cost Homomorphisms to Semicomplete Multipartite Digraphs. Submitted to Discrete Applied Math.

[6] G. Gutin, A. Rafiey, A. Yeo and M. Tso, Level of repair analysis and minimum cost homomorphisms of graphs. Discrete Appl. Math. 154 (2006), 881-889.

[7] M. M. Halldorsson, G. Kortsarz, and H. Shachnai, Minimizing average completion of dedicated tasks and interval graphs. Approximation, Randomization, and Combinatorial Optimization (Berkeley, Calif, 2001), Lecture Notes in Computer Science, vol. 2129, Springer, Berlin, 2001, pp. 114-126. 
[8] P. Hell, Algorithmic aspects of graph homomorphisms, in 'Survey in Combinatorics 2003', London Math. Soc. Lecture Note Series 307, Cambridge University Press, 2003, 239 - 276.

[9] P. Hell and J. Nešetřil, On the complexity of $H$-colouring. J. Combin. Theory B 48 (1990), $92-110$.

[10] P. Hell and J. Nešetřil, Graphs and Homomorphisms. Oxford University Press, Oxford, 2004.

[11] K. Jansen, Approximation results for the optimum cost chromatic partition problem. $J$. Algorithms 34 (2000), 54-89.

[12] T. Jiang and D.B. West, Coloring of trees with minimum sum of colors. J. Graph Theory 32 (1999), 354-358.

[13] S. Khanna, M. Sudan, L. Trevisan and D. Williamson, The approximability of constraint satisfaction problems. SIAM J. Comput. 30 (2000), 1863-1920.

[14] L.G. Kroon, A. Sen, H. Deng, and A. Roy, The optimal cost chromatic partition problem for trees and interval graphs, Graph-Theoretic Concepts in Computer Science (Cadenabbia, 1996), Lecture Notes in Computer Science, vol. 1197, Springer, Berlin, 1997, pp. 279-292.

[15] K. Supowit, Finding a maximum planar subset of a set of nets in a channel. IEEE Trans. Computer-Aided Design 6 (1987), 93-94. 